OPEN ACCESS

Edited by:

Valentina Tomassini, Cardiff University, United Kingdom

Reviewed by: Niels Hellings,

University of Hasselt, Belgium Nicolas Fissolo,

Vall d'Hebron Research Institute (VHIR), Spain

*Correspondence: Sara Gil-Perotin sara.garcia@uv.es

Bonaventura Casanova csurem_hlf@gva.es

Specialty section

This article was submitted to

Multiple Sclerosis and

Neuroimmunology,

a section of the journa

Frontiers in Neurology

Received: 11 May 2019 Accepted: 04 September 2019 Published: 23 September 2019

Citation:

Gil-Perotin S, Castillo-Villalba J, Cubas-Nuñez L, Gasque R, Hervas D, Gomez-Mateu J, Alcala C

Perez-Miralles F, Gascon F,

Dominguez JA and Casanova $B$ (2019) Combined Cerebrospinal Fluid Neurofilament Light Chain Protein and Chitinase-3 Like-1 Levels in Defining

Disease Course and Prognosis in Multiple Sclerosis.

Front. Neurol. 10:1008. doi: 10.3389/fneur.2019.01008

\section{Combined Cerebrospinal Fluid Neurofilament Light Chain Protein and Chitinase-3 Like-1 Levels in Defining Disease Course and Prognosis in Multiple Sclerosis}

\author{
Sara Gil-Perotin ${ }^{1,2 *}$, Jessica Castillo-Villalba ${ }^{1,2}$, Laura Cubas-Nuñez ${ }^{1,2}$, Raquel Gasque ${ }^{2}$, \\ David Hervas ${ }^{3}$, Josep Gomez-Mateu ${ }^{4}$, Carmen Alcala ${ }^{1}$, Francisco Perez-Miralles ${ }^{1}$, \\ Francisco Gascon ${ }^{5}$, Jose Andres Dominguez ${ }^{5}$ and Bonaventura Casanova ${ }^{1,2 *}$

\begin{abstract}
${ }^{1}$ Multiple Sclerosis Unit, Hospital Universitari i Politècnic La Fe, Valencia, Spain, ${ }^{2}$ Research Group in Neuroimmunology, Health Research Institute La Fe, Valencia, Spain, ${ }^{3}$ Biostatistics Unit, Health Research Institute La Fe, Valencia, Spain, ${ }^{4}$ Neurology Department, Hospital Universitari Dr. Peset, Valencia, Spain, ${ }^{5}$ Neuroimmunology Unit, Hospital Clínic de
\end{abstract} \\ València, Valencia, Spain
}

Background: Neurofilament light chain protein (NFL) and chitinase3-like1 (CHI3L1) have gained importance recently as prognostic biomarkers in multiple sclerosis (MS).

Objectives: We aimed to investigate NFL and CHI3L1 cerebrospinal fluid (CSF) profiles in multiple sclerosis and the informative and prognostic potential of the individual and combined measures.

Methods: CSF NFL and CHI3L1 levels were measured in a cross-sectional cohort of 157 MS patients [99 relapsing-remitting (RRMS), 35 secondary progressive (SPMS), and 23 primary progressive (PPMS)]. Clinical relapse and/or gadolinium-enhanced lesions (GEL) in MRI within 90 days from CSF collection by lumbar puncture (LP) were registered and considered as indicators of disease activity. Longitudinal treatment and disability data were evaluated during medical visits with a median follow-up of 50 months.

Results: CSF levels of NFL and CHI3L1 were higher in MS patients compared to non-MS controls. In RRMS and SPMS patients, increased NFL levels were associated with clinical relapse, and gadolinium-enhanced lesions in MRI $(p<0.001)$, while high $\mathrm{CHI}$ L1 levels were characteristic of progressive disease $(p=0.01)$. In RRMS patients, CSF NFL, and CHI3L1 levels correlated with each other $(r=$ 0.58), and with IgM-oligoclonal bands ( $p=0.02$ and $p=0.004$, respectively). In addition, CSF CHI3L1 concentration was a predictor for 1-point EDSS worsening $\{\mathrm{HR}=2.99[95 \% \mathrm{Cl}(1.27,7.07)]\}$ and progression during follow-up $\{\mathrm{HR}=18$ $[95 \% \mathrm{Cl}(2.31,141.3)]\}$. The pattern of combined measure of biomarkers was useful to discriminate MS phenotypes and to anticipate clinical progression: RRMS more frequently presented high NFL combined with low CHI3L1 levels, compared to SPMS (HR 0.41 [0.18-0.82]), and PPMS (HR 0.46 [0.19-0.87]), while elevation of both biomarkers preceded diagnosis of clinical progression in RRMS patients (log rank $=0.02$ ). 
Conclusions: Individual measures of CSF NFL and CHI3L1 are biomarkers of disease activity and progression, respectively. The pattern of combined measure discriminates MS phenotypes. It also predicts the subset of RRMS patients that will progress clinically allowing early intervention.

Keywords: YKL-40, CHI3L1, NFL, progressive multiple sclerosis, gadolinium-enhancing lesions

\section{INTRODUCTION}

Neurofilament light protein (NFL), a cytoskeletal polypeptide of the axon (1), and chitinase 3-like 1 (CHI3L1- also known as YKL40 or gp39), a glycoprotein secreted by activated glia in the central nervous system (CNS) (2), have both shown to be biomarkers of axonal destruction, and inflammation in multiple sclerosis (MS), respectively.

NFL is not a specific biomarker but a reflection of axonal destruction in several neurological diseases (3-6). In MS, cerebrospinal fluid (CSF), and serum levels of NFL have been suggested as markers for disease activity in MS (7) and predictors of clinically isolated syndrome (CIS) conversion to MS (8-11). NFL is also considered as a prognostic marker of worse outcomes regarding brain atrophy $(12,13)$ and disability progression (13). Its levels in serum and CSF decrease with Disease-Modifying Therapies (DMT), therefore, it seems to be a good surrogate marker for measuring response to treatment (14-18).

CHI3L1 expression is not restricted to the CNS, but its presence in the CSF has been related to endogenous secretion by astrocytes, and microglia/monocytes (19). Its increase has been related to CIS conversion to MS (20, 21), advanced (20), and/or progressive disease (7, 22-24), cognitive impairment (25), and increased disability $(20,24)$. Like NFL, CHI3L1 levels were responsive to DMTs in relapsing MS (15, 26-28).

In relapsing-remitting MS (RRMS), the presence of CSF oligoclonal IgM bands (OCMB) has been associated with more substantial T2 lesion load, increased gadolinium-enhancing lesions (GEL), higher relapse rate, more neurological disability, and brain volume loss over time. OCMB have also been related to earlier conversion to secondary progressive MS (SPMS) and can help to identify a subset of primary progressive MS (PPMS) patients with a more inflammatory phenotype (29-31).

Although literature regarding the role of NFL and CHI3L1 in MS is profuse, there is limited information about their relationship with OCMB and little is known about the diagnostic and predictive role of the combined assessment of CSF levels of both NFL and CHI3L1. In this work, with a cohort of 157 patients including RRMS and progressive phenotypes (SPMS; PPMS) and with prospectively collected disability data, we aimed to demonstrate that the combined measure of both biomarkers in the CSF might have value, not only in identifying distinct MS phenotypes but also in predicting accrual of disability and further diagnosis of progressive disease in RRMS patients.

\section{METHODS \\ Study Cohort}

The study included all MS patients with available samples of CSF, magnetic resonance imaging (MRI), and longitudinal disability data, seen at two university hospitals in Valencia, Spain (Hospital Universitari I Politècnic La Fe and Hospital Clinic Universitari) between 2008 and 2017 (Figure 1). Demographic and clinical data were retrospectively collected with the last update in December 2018. All patients provided written informed consent. Non-MS Control CSF samples were selected from patients who were seen because of headache with fever, Pseudotumor cerebri, encephalopathy, or dementia [median age: 33 (IQR, 29-35); 59\% female]. All patients in this cohort had a normal brain MRI and CSF analysis, with no evidence of infection, inflammation, autoimmunity, or known neurodegenerative disease. The study was approved by the Institutional Ethics Committee in Hospital Universitari I Politècnic La Fe (reference number PI17/01544).

\section{Definitions}

Diagnosis of clinically definite MS was made according to 2017 McDonald criteria (32). Active disease was considered when a clinical attack occurred and/or at least one gadolinium-enhanced lesion (GEL) was present in T1-weighted MRI. A clinical attack or relapse was defined as an acute worsening of neurologic function lasting more than $24 \mathrm{~h}$, not explained by fever or physical stress, and followed by a variable degree of recovery. Urinary symptoms alone were not considered for a diagnosis of relapse. CSF samples were considered contemporary to active disease when a lumbar puncture (LP) was performed within 90 days after assessment of clinical attack and/or GEL. Clinical phenotypes were classified according to modified Lublin criteria (33). A secondary progressive phase of multiple sclerosis (SPMS) was considered when patients with an EDSS score $\geq 3.0$ had a 6month confirmed increase to an EDSS score of $\geq 4.0$, pyramidal functional system was $\geq 2.0$, and there was no evidence of relapse. PPMS phenotype was assigned to those patients who fulfilled 2017 McDonald criteria for PPMS (34). Neurological disability was defined as the neurological worsening, derived not only from clinical relapse but also from accrual of neurological symptoms in the absence of relapse or radiological signs of acute inflammation. It was estimated according to the expanded disability status scale (EDSS) (35) at the time of LP and every 6 months until the last visit. One-point EDSS worsening was considered anytime during follow-up as a measure of neurological disability, secondary to acute inflammation, or to progression-related accrual of 

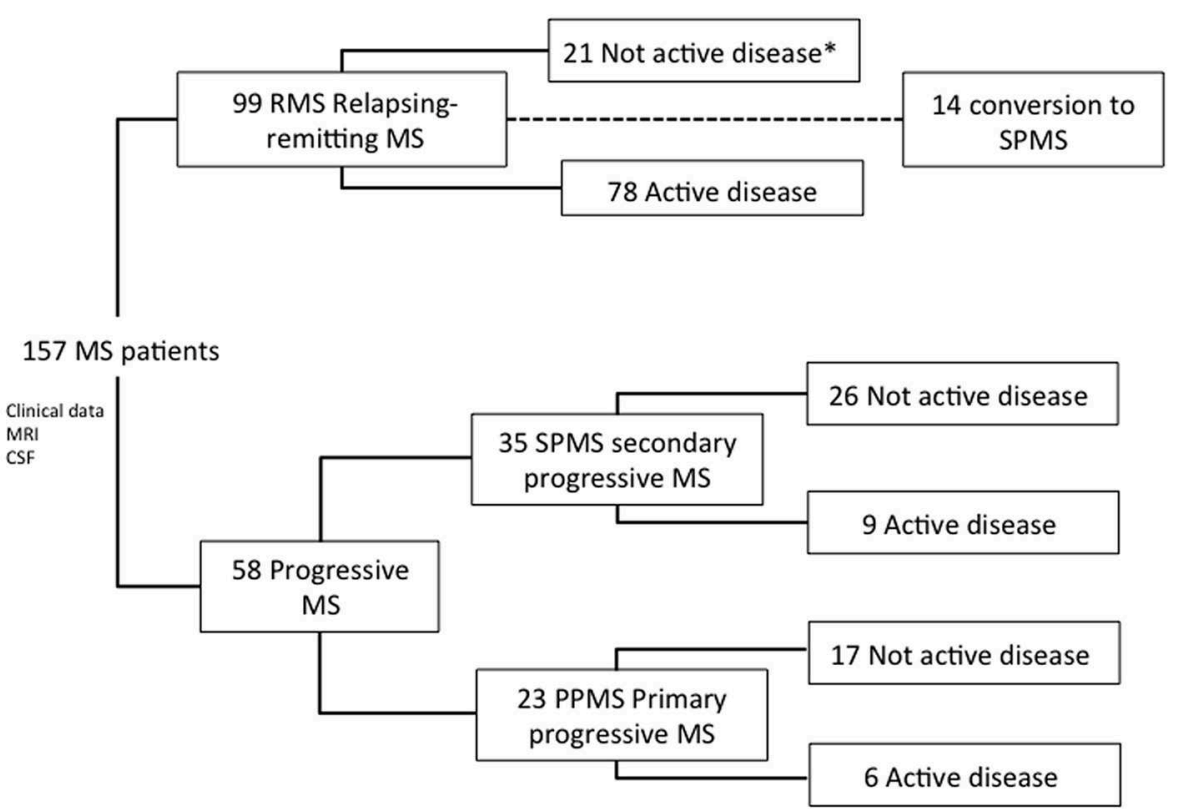

*Activity determined by clinical relapses and/or MRI activity (contrast-enhancing lesions) within 90 days from LP collection

FIGURE 1 | Flow chart of study cohort.

disability. Treatment failure was considered as a loss of NEDA3 status (a composite measure of disease activity based on the absence of relapses, no evidence of disability progression by the EDSS, and no new T2 lesions or GEL in MRI) (36).

\section{Treatment}

Patients with clinically definite MS were treated with first-line DMT, chosen at physician discretion, unless one of the following circumstances occurred: (i) two clinical attacks in 1 year, (ii) a clinical attack and/or a new GEL within 3 months after the bout, (iii) a disabling clinical attack with residual EDSS of at least 2 points. In these cases, and those with treatment failure, second line DMT was administered. Non-responders to first- and second line DMT proceeded to autologous stem cell transplantation (ASCT).

\section{CSF Sampling and Biomarker Analysis}

$\mathrm{CSF}$ samples were stored at $-80^{\circ} \mathrm{C}$ in the Biobank $\mathrm{La} \mathrm{Fe}$ with the approval of the Ethics and Scientific Committees (PT17/0015/0043). NFL and CHI3L1 levels in CSF were assessed by enzyme-linked immunosorbent assays using commercially available kits according to manufacturer's instructions (Uman Diagnostics AB, Umea, Sweden and Quantikine ELISA kit, R\&D Systems, respectively). The mean intra-assay coefficients of variation for NFL and CHI3L1 were 4.5 and $6.5 \%$, and inter-assay 3.3 and $5.2 \%$, respectively.

\section{Other Ancillary Tests}

Brain and spinal cord 1.5/3.0 Tesla MRI were performed at diagnosis and during the follow-up. CSF oligoclonal IgG bands and oligoclonal IgM bands (OCMB) against lipids were studied by isoelectric focusing and immunoblotting, as previously described (29). Serum and CSF were tested to rule out infections, other inflammatory diseases, and non-MS demyelinating diseases.

\section{Statistical Analysis}

Statistical analysis was performed by the Biostatistics Department of the Research Health Institute La Fe. Categorical variables were described by counts (n) and percentages (\%), continuous, and ordinal variables by median (first and third quartiles). CSF NFL/CHI3L1 levels were log-transformed (logNFL and $\log \mathrm{CHI}$ (2L1) to meet the normality assumption. Comparisons between groups were performed with U-Mann Whitney, Student $t$-test, and a one-way analysis of covariance (ANCOVA) using age, sex, and disease duration as covariates, with no significant changes in the estimated effects found for NFL, and CHI3L1. Bonferroni post-hoc correction method was performed for multiple comparisons. Correlations were analyzed with the Pearson's product-moment correlation test. Spearman's rank correlation method was additionally performed as a sensitivity analysis. To analyze the contribution of paired values of biomarkers, patients were categorized into four groups with a median split method (the median value was calculated from the RRMS cohort without disease activity): group 1 included patients with CSF NFL and CHI3L1 levels below median values, group 2 and 3 included patients with only NFL or CHI3L1 values above median, respectively, and group 4 comprised patients with CSF NFL, and CHI3L1 concentrations above median levels. Bivariate analysis with Kaplan Meier survival curves was performed for the probability of 1-point EDSS worsening, diagnosis of 
TABLE 1 | Demographics and CSF findings.

\begin{tabular}{|c|c|c|c|c|}
\hline Variable & RRMS ( $n=99)$ & SPMS $(n=35)$ & PPMS $(n=23)$ & $p$ \\
\hline Age at disease onset (years) & $28(23.5,36.5)$ & $29(23,36)$ & $41(34.5,47.5)$ & $<0.001$ \\
\hline Age at time of LP (years) & $35(29.5,41)$ & $45(38.5,50)$ & $51(41,56)$ & $<0.001$ \\
\hline Female gender & 79 (79.8\%) & $21(60 \%)$ & $10(43.5 \%)$ & $<0.001$ \\
\hline Time from diagnosis to LP (years) & $2.1(0.3,9.8)$ & $15.8(8.8,19.3)$ & $8.3(5.0,11.8)$ & $<0.001$ \\
\hline Follow-up from LP (years) & $4.4(3.0,5.9)$ & $4.3(2.3,5.7)$ & $3.1(2.5,5.7)$ & $<0.001$ \\
\hline Clinical relapse at time of LP & $53(53.5 \%)$ & $2(5.88 \%)$ & $1(5 \%)$ & $<0.001$ \\
\hline GEL in MRI at time of LP & $53(56.4 \%)$ & $8(25.81 \%)$ & $6(30 \%)$ & $<0.001$ \\
\hline OCB-lgG & 91 (91.9\%) & $31(88.6 \%)$ & 22 (95.7\%) & 1 \\
\hline OCB-lgM & 49 (49.5\%) & 18 (51.4\%) & $10(43.5 \%)$ & 0.9 \\
\hline Baseline EDSS & $2(1,3)$ & $5.5(4.25,6.5)$ & $5(3.5,6)$ & $<0.001$ \\
\hline NFL in CSF (pg/ml) & $591.9(290,1106)$ & $533(266,678)$ & $450(304,746)$ & 0.6 \\
\hline CHI3L1 in CSF (ng/ml) & $118.97(81,186)$ & $139.55(96,212)$ & $180.25(146,265)$ & 0.01 \\
\hline
\end{tabular}

RRMS, relapsing-remitting multiple sclerosis; SPMS, secondary progressive multiple sclerosis; PPMS, primary progressive multiple sclerosis; LP, Iumbar puncture; GEL, gadolinium-

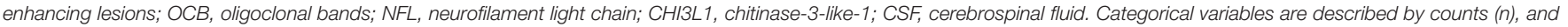

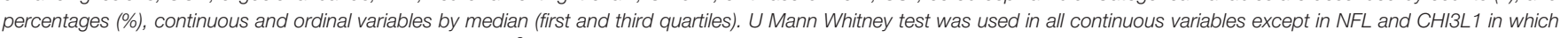
logNFL and logCHI3L1 were analyzed with T-student test. $\chi^{2}$ test was used for categorical variables.

progressive disease, escalation of treatment, and occurrence of first relapse after LP in RRMS patients. Predictors were age, sex, disease activity, EDSS, $\operatorname{logNFL}$, and $\log \mathrm{CHI} 3 \mathrm{~L} 1$ (or alternatively patient's biomarker categories), presence of OCMB in the CSF, and treatment at the time of LP. Elastic net penalized Cox proportional hazard regression models were performed in the RRMS subgroup for the same outcomes and predictors. After the selection of the predictors with the elastic net algorithm, the model was refitted with a standard Cox regression to get approximate $p$-values and estimates of the effects. Additionally, a multinomial logistic regression model was performed including log-transformed CSF NFL, and CHI3L1 levels as predictors. MS clinical phenotypes (RRMS, SPMS, and PPMS) and diagnosis of SPMS were used as response variables with RRMS as the reference group. A plot of marginal effects was drawn to ease the interpretation of multinomial logistic regression model. All analyses were conducted using SPSS $21.0 \mathrm{v}$ and $\mathrm{R}$ version 3.4.3 (The CRAN project).

\section{RESULTS}

\section{Patient Characteristics}

We studied 157 MS patients, 99 RRMS (63\%), and 58 progressive MS (37\%), of which 35 were SPMS (22\%), and 23 PPMS (15\%) (Figure 1).

Clinical and demographic features are shown in Table 1. Clinical phenotypes differed in age at disease onset and at time of LP, time lapse between disease onset and LP, gender, disease activity (relapse or/and GEL), and EDSS. The median follow-up time after LP was also significantly different between groups. In 28 patients, LP was performed within 90 days after disease onset.

Ninety-three patients (60\%) had documented clinical attack and/or GEL in the MRI within 90 days from LP. Median time from a clinical attack to LP and from MRI to CSF collection was 38 days $(14,65)$ and 22 days $(5,50)$, respectively. At the time of analysis, 14 patients in the RRMS cohort had sustained an increase in disability without clinical attacks and, therefore, were reassigned to SPMS phenotype. Disease duration in these patients was significantly higher compared to that in patients who did not progress during follow-up $(p=0.03)$.

\section{CSF Levels of NFL and CHI3L1 in MS and Relationship With Disease Inflammatory Activity or Disease Course}

Overall, the median CSF levels of NFL were $536 \mathrm{pg} / \mathrm{ml}(289,880)$ in MS patients and $158 \mathrm{pg} / \mathrm{ml}(125,190)$ in non-MS controls $(p<$ $0.001)$. The distribution was highly dispersed in the three clinical MS forms with no significant differences between them $(p=0.6)$ (Table 1; Figure 2A). The median CSF levels of CHI3L1 were $133 \mathrm{ng} / \mathrm{ml}(93,215)$ in MS patients and $58 \mathrm{ng} / \mathrm{ml}(47,77)$ in nonMS controls $(p<0.001)$, and differed between MS phenotypes being $119 \mathrm{ng} / \mathrm{ml}(81,186)$ in RRMS, $139 \mathrm{ng} / \mathrm{ml}(95,211)$ in SPMS, and $180 \mathrm{ng} / \mathrm{ml}(146,265)$ in PPMS $(p=0.01)$ (Table 1; Figure 2A). Bonferroni post-hoc correction showed a significant difference between RRMS and PPMS ( $p=0.009$ ).

At the time of LP, 93 patients were considered active (78 RRMS, 9 SPMS, and 6 PPMS) according to the given definition of active disease (clinical attack and/or the presence of GEL in the MRI). CSF NFL levels in patients with and without active disease were $710 \mathrm{pg} / \mathrm{ml}(323,1480)$, and $329 \mathrm{pg} / \mathrm{ml}(237,614)$, respectively ( $p<0.001$; Figure $2 \mathrm{~B}$ ). This difference maintained statistical significance in RRMS $(p=0.016)$ and SPMS $(p=0.007)$ but not in PPMS $(p=0.265)$. Considering only the presence of GEL, NFL levels differed depending on whether GEL was present $(740 \mathrm{pg} / \mathrm{ml})(313,1,719)$ or absent $(385 \mathrm{pg} / \mathrm{ml})(263,710)(p$ $<0.001$ ), but there was no evidence of a correlation with the number of this type of lesions $(p=0.395)$.

Median CSF CHI3L1 levels in active patients were $133 \mathrm{ng} / \mathrm{ml}$ $(85,217)$, which was not significantly different from patients without inflammatory activity $[140 \mathrm{ng} / \mathrm{ml}(93,220)](p=0.862$; Figure 2). Irrespective of the MS phenotype was $(p=0.645)$, 
A

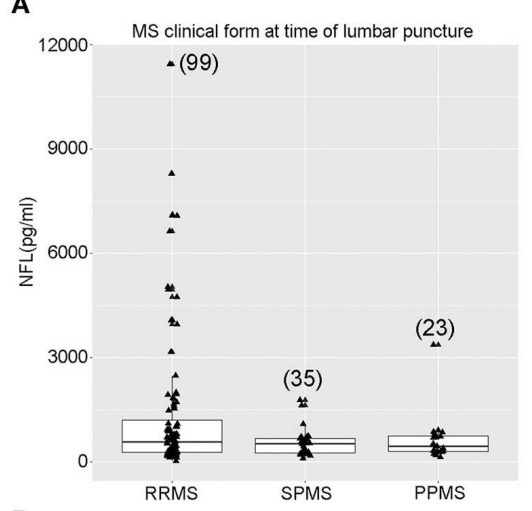

B

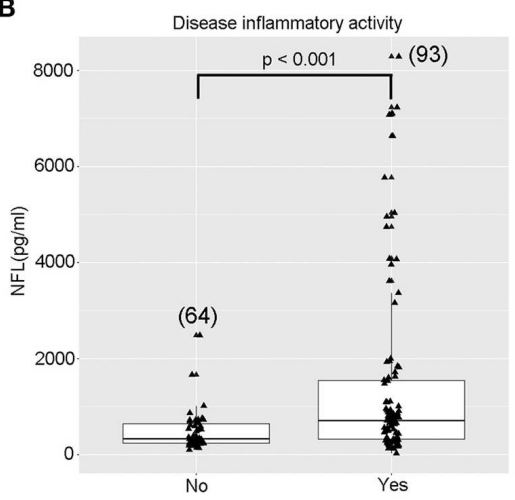

C

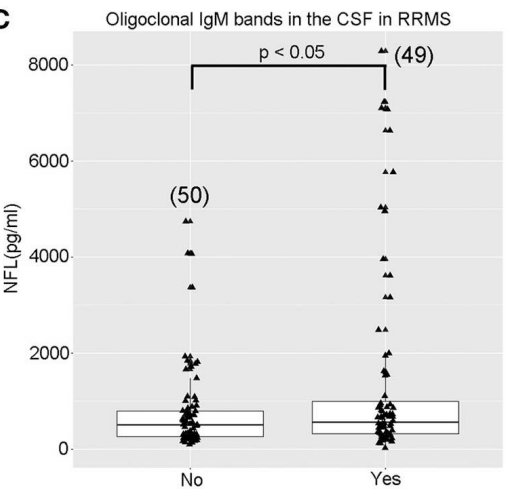

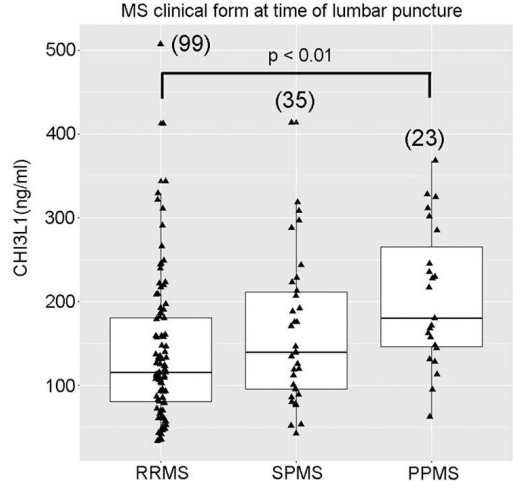
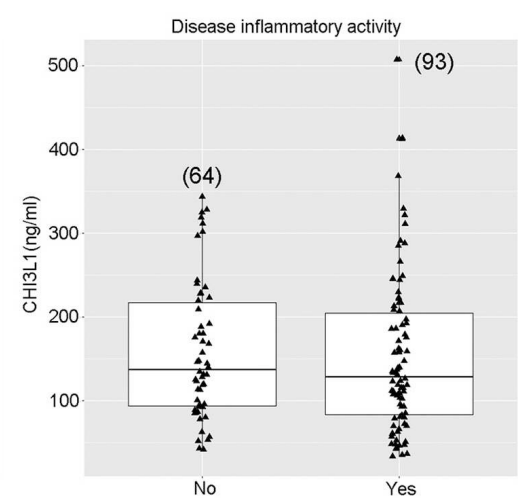

Oligoclonal IgM bands in the CSF in RRMS

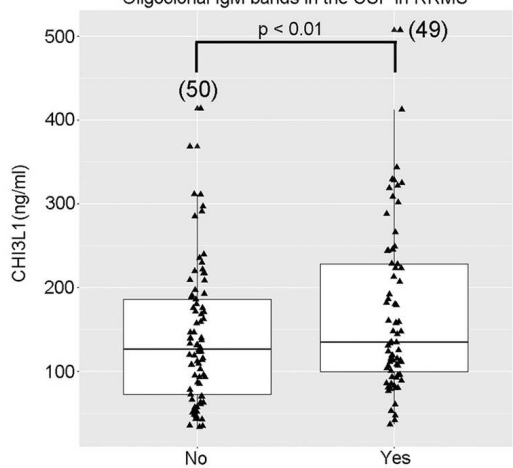

D
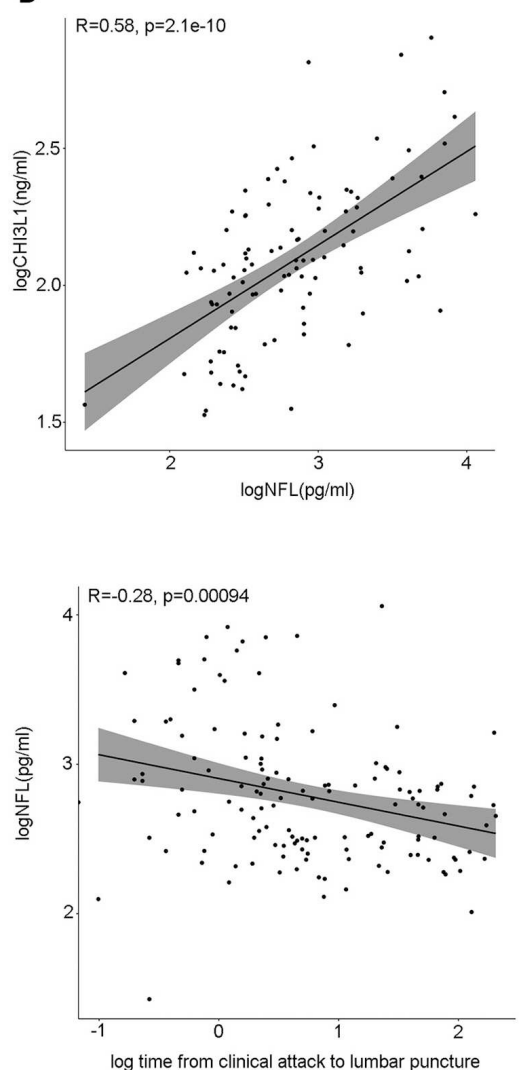

FIGURE 2 | CSF levels of NFL and CHI3L1 in MS in association to phenotype, inflammatory activity, and other influencing variables. (A) Patients with disease activity had higher CSF NFL levels compared to patients without documented disease activity (defined as clinical attack and/or GEL in the MRI within 90 days of CSF collection) (Student $T$-test $p<0.001$ ). CHI3L1 levels were not different with regards to disease activity (Student $T$-test $p=0.705)$. (B) NFL levels were not different between MS forms (ANOVA, $p=0.6$ ) while CHI3L1 were increased in progressive MS compared to RRMS (ANOVA $p=0.01$; Bonferroni post-hoc RRMS vs. PPMS $p$ $=0.009$ ). (C) Both NFL and CHI3L1 were higher in the presence of OCMB in the RRMS cohort (Student $T$-test $p=0.02$ and $p=0.004$, respectively). (D) NFL and CHI3L1 correlated between each other (Pearson's $r=0.58 ; p<0.001$ ) in the RRMS cohort. NFL levels were lower as time from clinical relapse passed (Pearson's $r=$ $0.29 ; p=0.04)$. Sample size for each condition is expressed between brackets in the diagram. Student $T$-test and correlations were performed with log-transformed NFL and CHI3L1. NFL, neurofilament light chain; CHI3L1, chitinase-3-like-1; RRMS, relapsing-remitting multiple sclerosis; SPMS, secondary progressive multiple sclerosis; PPMS, primary progressive multiple sclerosis. GEL, gadolinium-enhanced lesions; CSF, cerebrospinal fluid; MRI, magnetic resonance imaging.

SPMS $(p=0.390)$, or PPMS $(p=0.267)$. CSF CHI3L1 levels in patients with or without GEL were not significantly different, being $133 \mathrm{ng} / \mathrm{ml}(87,219)$ and $135 \mathrm{ng} / \mathrm{ml}(88,196)(p=0.705)$, and the number of GEL did not correlate with CHI3L1 levels $(p=$ 0.395). Although, overall, median levels of CSF NFL, and CHI3L1 were high in MS, extreme values of both biomarkers were characteristic of RRMS patients with active disease (Figure 2B).

\section{Correlation of CSF NFL and CHI3L1 Levels With Other Clinical Variables}

There was a significant correlation between CSF CHI3L1 and NFL concentrations ( $r=0.46 ; p<0.001$; Figure 2D) but, when analyzing this correlation in distinct MS clinical forms it only persisted in RRMS $(r=0.58 ; p<0.001)$ compared to SPMS $(r$ $=0.15 ; p=0.195)$, and PPMS $(r=0.3 ; p=0.081)$. There was 
a trend between decreased CSF NFL levels and disease duration at the time of LP $(r=-0.2 ; p=0.02)$ or time from clinical relapse $(r=-0.28 ; p=0.04)$. NFL was higher in the presence of OCMB (652.0 pg/ml vs. $487.8 \mathrm{pg} / \mathrm{ml} ; p=0.02)$ only in the RRMS subgroup of patients (Figure 2D).

Higher concentrations of CSF CHI3L1 were associated with increased age $(r=0.2 ; p=0.013)$ and higher EDSS at the time of LP $(r=0.21 ; p=0.009)$, although correlations were weak. The presence of OCMB was more frequent with higher levels of CHI3L1 only in the RRMS subgroup $(125 \mathrm{ng} / \mathrm{ml}$ vs. $108 \mathrm{ng} / \mathrm{ml} ; p=0.004$ ), as it occurred with NFL (Figure 2C). OCMB was not associated to disease activity if we considered a clinical attack $\left(\chi^{2} ; p=0.132\right)$ or presence of GEL $\left(\chi^{2} ; p=\right.$ 0.519 ) separately, but patients with both conditions had OCMB in the CSF more frequently $\left(\chi^{2} ; p=0.034\right)$. The mean number of GEL in the presence of OCMB was 2.53 , while in its absence it was ere 1.36, but this observation did not reach statistical significance $(p=0.121)$.

At the time of LP, 109 patients were not treated, and 49 patients were treated (26 RRMS, 20 SPMS, 3 PPMS). Twenty-six patients were under first-line DMT (23 IFN-beta, 3 glatiramer acetate), 20 were on second-line DMT (2 fingolimod, 15 natalizumab, 3 antiCD20), and the remaining 3 had undergone ASCT in the previous 5 years after treatment failure. Median CSF NFL and CHI3L1 levels did not differ between patients being treated at time of LP $(576 \mathrm{pg} / \mathrm{ml}$ and $135 \mathrm{ng} / \mathrm{ml})$ and untreated patients $(514 \mathrm{pg} / \mathrm{ml}$ and $123 \mathrm{ng} / \mathrm{ml} ; p=0.8$ and $p=$ 0.2 , respectively).

\section{Predictive Value of CSF NFL and CHI3L1 in RRMS}

After LP, our cohort was prospectively followed and clinical and disability data, including relapse rate, and date of conversion to progressive disease, were registered. Twenty-five RRMS patients (25\%) experienced a 1-point increase in disability assessed with EDSS after LP. Bivariate Kaplan Meier analysis showed CHI3L1 ( $\log \operatorname{rank} p=0.018$ ) as potential predictor with a tendency for NFL ( $\log \operatorname{rank} p=0.0523)$ and disease activity $(\log \operatorname{rank} p=$ 0.109 ) to differ in 1-point EDSS worsening. After multivariable analysis, only CHI3L1 persisted as independent predictor $\{\mathrm{HR}=$ $2.99[95 \%$ CI $(1.27,7.07)]\}$. NFL had no impact on this outcome (Table 2).

Fourteen RRMS patients (14\%) progressed to SPMS. Bivariate Kaplan Meier analysis detected CHI3L1 $(\log$ rank $p=0.0001)$, NFL $(\log \operatorname{rank} p=0.015)$, and disease activity (log rank $p$
$=0.001)$ as potential predictors for diagnosis of SPMS. With Cox analysis, only higher CHI3L1 levels, $\{\mathrm{HR}=18[95 \% \mathrm{CI}$ $(2.31,141.3)]\}$, and active disease $\{\mathrm{HR}=0.186$ [95\% CI $(0.06$, $0.62)]\}$ were shown to be associated with disease progression after LP (Table 2). NFL was not independent predictor of disease progression.

Overall, escalation to second line DMT was needed in 57 patients during follow-up ( 9 fingolimod, 29 natalizumab, 14 rituximab, 2 ASCT, 1 alemtuzumab, 2 metotrexate). Bivariate Kaplan Meier analysis found gender $(\log \operatorname{rank} p=0.043)$, and EDSS $(\log \operatorname{rank} p=0.047)$ to be potential predictors for the need of treatment escalation. None had predictive value for this outcome after multivariate analysis.

Forty-two RRMS patients (42\%) had a clinical relapse after LP. Bivariate Kaplan Meier analysis detected gender $(\log \operatorname{rank} p=$ 0.043 ) and EDSS ( $\log \operatorname{rank} p=0.046)$ as potential predictors for clinical relapse. None had predictive value for this outcome after multivariate analysis.

\section{Combined Assessment of CSF NFL and CHI3L1 Concentrations in Distinct MS Phenotypes}

With the purpose of investigating the value of the combined measure in MS prognosis, we stratified patients in four groups according to both NFL and CHI3L1 values with respect to their median in the RRMS cohort in the absence of disease activity (Group 1: both NFL and CHI3L1 below median value; Group 2: high NFL; Group 3: High CHI3L1; Group 4: NFL and CHI3L1 above median values). Bivariate analysis using biomarker profiles as predictors showed that being a patient in Group 4 was predictive of diagnosis of progressive disease during follow-up ( $\log$ rank $p=0.02$ ) compared to being included in any of the other groups of patients (Figure 3). Paired biomarker levels were not predictive of increasing 1-point in disability ( $\log$ rank $p=$ $0.094)$, need for escalation therapy $(\log \operatorname{rank} p=0.173)$ or relapse after LP $(\log$ rank $p=0.783)$.

The representation of paired values NFL/CHI3L1 of biomarkers (each pair corresponding to one patient), in density maps with a raster diagram, showed that peak density of patients was distinctly distributed between non-MS controls and MS patients, and within MS, between distinct MS clinical forms (Figure 4). RRMS patients that were diagnosed of SPMS during follow-up corresponded to patients with both NFL and CHI3L1 concentrations above median values.

TABLE 2 | Cox regression analyses for 1-point EDSS worsening and diagnosis of progressive disease in RRMS patients during follow-up.

\begin{tabular}{|c|c|c|c|c|c|c|}
\hline & Estimate & Std. error & HR & Lower $95 \%$ & Upper $95 \%$ & $p$-value \\
\hline \multicolumn{7}{|c|}{ 1-point EDSS worsening } \\
\hline $\log _{10}(\mathrm{CHI} 3 \mathrm{~L} 1)$ & 1.097 & 0.438 & 2.996 & 1.269 & 7.074 & 0.008 \\
\hline \multicolumn{7}{|c|}{ Disease progression } \\
\hline $\log _{10}(\mathrm{CHI} 3 \mathrm{~L} 1)$ & 2.893 & 1.050 & 18.044 & 2.305 & 141.3 & 0.0002 \\
\hline Disease activity & -1.682 & 0.607 & 0.186 & 0.057 & 0.611 & 0.002 \\
\hline
\end{tabular}

CHI3L1, chitinase-3-like-1. 


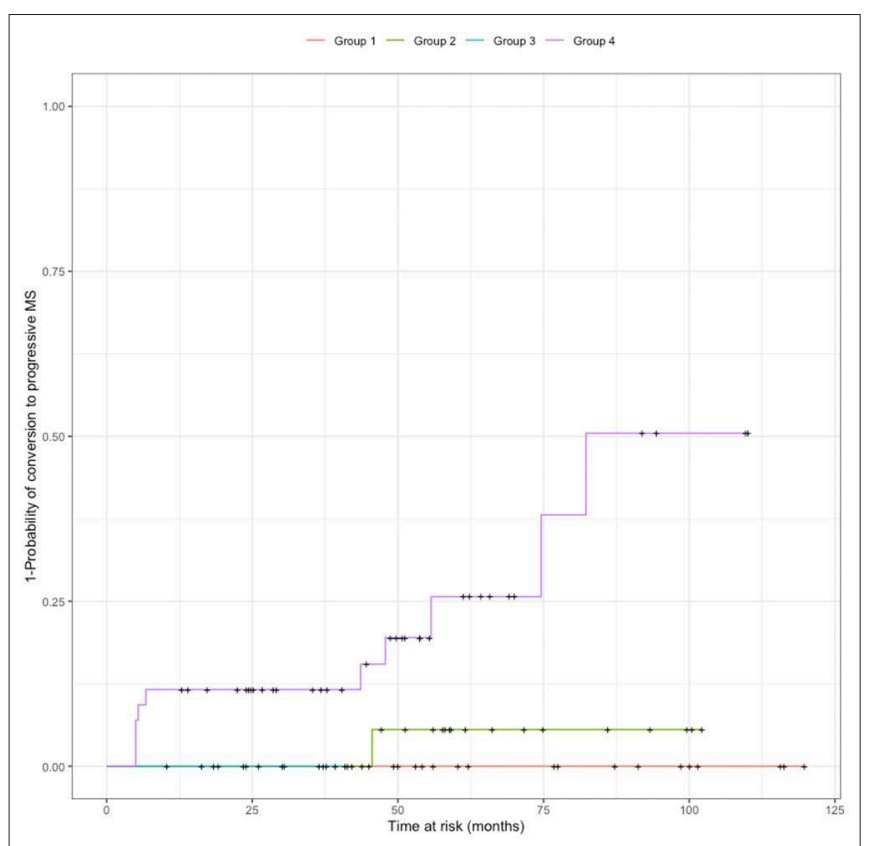

FIGURE 3 | Probability of conversion to SPMS according to biomarker profile. Patients were cathegorized according to NFL and CHI3L1 median values (calculated in the RRMS cohort without disease activity) into four groups: Group 1: both NFL and CHI3L1 below median value; Group 2: high NFL; Group 3: High CHI3L1; Group 4: NFL and CHI3L1 above median values. Patients included in group 4 had more probability to be diagnosed of SPMS during follow-up than patients pertaining to the other groups (log rank $p=0.02)$. SPMS: secondary progressive multiple sclerosis.

We additionally tested the interaction between both biomarkers to discriminate MS phenotypes and predict progression using a multinomial logistic regression model (Table 3). The analysis showed a discriminating value of individual measures of CSF NFL and CHI3L1 between RRMS and SPMS [HR $0.41(0.18-0.82)$ ] and between RRMS and PPMS [HR 0.46 (0.19-0.87)]. An interaction between NFL and CHI3L1 in the multinomial regression model did not show statistical power to predict diagnosis of SPMS $\{0.96$ [95\% CI $(0.54,1.63)]\}$. However, the representation of these data in a marginal effects plot showed that the probability of switching to progressive disease was characteristic of patients with higher concentrations of CSF NFL and CHI3L1 (Figure 5C). MS phenotypes corresponding to low and median NFL levels are depicted in Figures 5A,B.

\section{DISCUSSION}

This study shows that MS clinical phenotypes and disease activity are associated with CSF NFL-CHI3L1 levels when assessed both individually and combined. NFL levels alone seemed to be more indicative of disease activity, whereas CHI3L1 levels were related to progression of disability as previously shown. As a novelty, here we state that both biomarkers in combination could be helpful to classify MS patients into distinct clinical forms, and to predict diagnosis of SPMS in the RRMS cohort.

We confirmed previous reports in which NFL levels were higher in MS patients compared to non-MS-controls, and further increased when clinical attack and/or GEL occurred (37). As time from relapse passed, CSF NFL levels decreased to median values, reinforcing the relationship between the NFL peak level, and acute axonal damage. We did not find a correlation of NFL with age, as it had been previously reported (8), probably because there were two opposing phenomena occurring in parallel: the disease activity being higher in younger individuals (38) and the ageassociated axonal degeneration or neurological co-morbidities associated with older individuals (39). This is why we did not use age-stratified reference values extracted from a non-MS cohort. Instead, we used median values from the RRMS cohort without disease activity as the threshold, and ANCOVA analysis was performed to adjust all our comparisons by age, gender, and disease duration.

CSF CHI3L1 levels were also high in MS compared to nonMS controls as previously reported $(19,40)$. Recent studies showed that CSF CHI3L1 levels were increased in progressive MS (24). Although median values of CSF CHI3L1 in MS were not related to disease activity, an observation also reported for plasma levels (24), some RRMS patients with active disease and particularly elevated CSF NFL concentration also presented increased CSF CHI3L1 levels (even 2-3 times the median values). To explore whether this increase was related to the disruption of the cerebrospinal fluid barrier during acute inflammation, the presence, and the number of GEL were both assessed, and correlation analyses were performed. We did not find an association between CSF CHI3L1 levels and presence of GEL, nor increasing numbers of GEL. This might imply that during a clinical relapse, other immunologic pathways than those causing GEL are responsible of CHI3L1 secretion. In fact, it has been demonstrated that an IL-13 pathway activation is related to high CHI3L1 levels (41) with differential patterns in bacterial or viral meningitis, suggesting the involvement of CHI3L1 in heterogeneous inflammatory pathways.

CSF CHI3L1 is also very dependent on age (40). Patients with progressive disease are usually older than RRMS patients, and therefore, the increase in CSF CHI3L1 levels could be merely explained by the abnormal priming of CNS glia that occurs in older ages [reviewed in (42)]. Although correlation of CHI3L1 with age was weak in our study, we adjusted all the analyses by age and disease duration to overcome the potential effect of age-dependent glial priming in our results.

OCMB is a known CSF biomarker of inflammatory disease and implies a worse prognosis in MS (31). In our cohort, its presence correlated with CSF NFL and CHI3L1 levels in RRMS patients, but we did not find an association with these biomarkers in progressive MS. This, and the fact that we found OCMB more frequently in patients with both clinical attack and GEL, suggests the more prominent role of this biomarker in acute inflammation than in chronic inflammation. OCMB positivity in our cohort was not able to predict disability accrual, diagnosis of progressive disease, occurrence of relapse, or treatment escalation. 


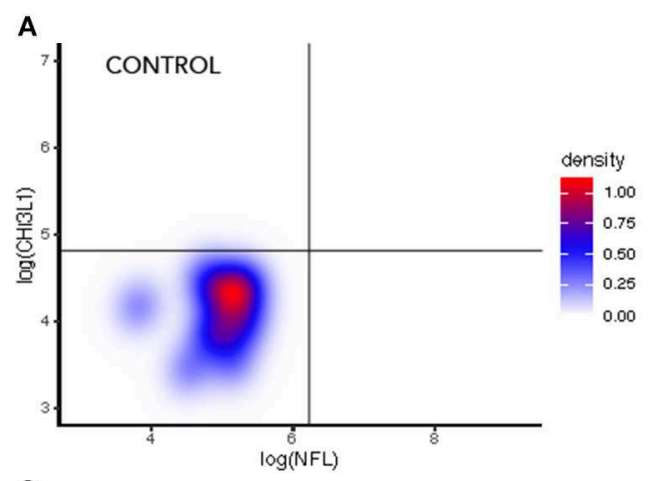

C

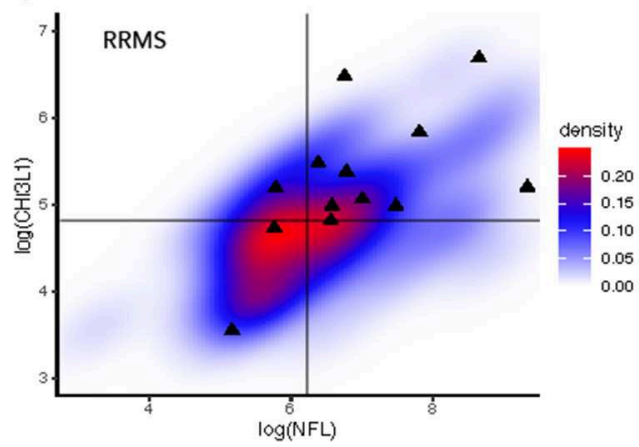

B

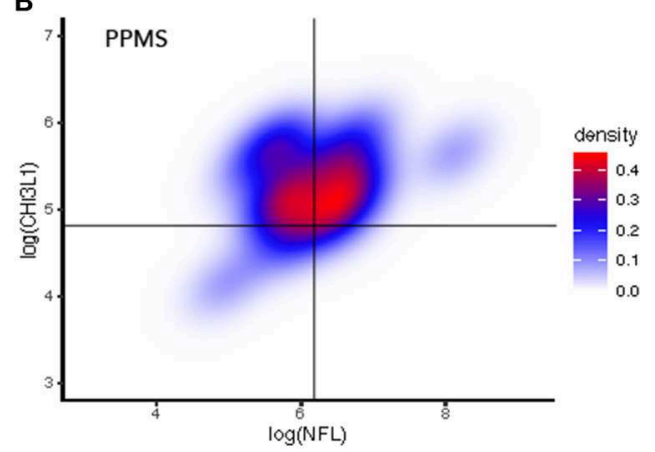

D

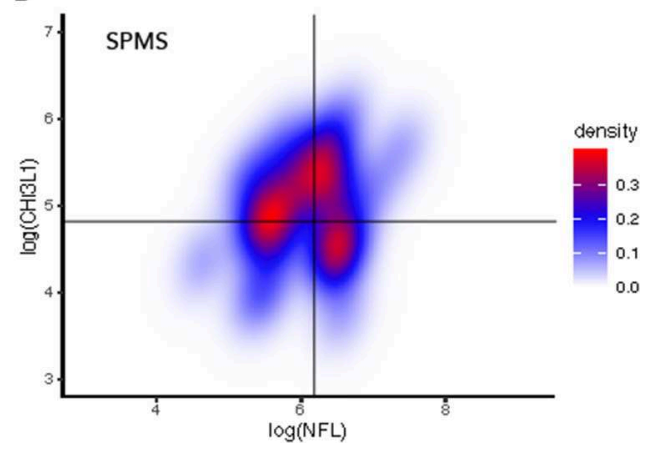

FIGURE 4 | Density maps representing paired NFL and CHI3L1 values in CSF of non-MS controls and MS patients. The black lines in each diagram represent the median log values of CSF NFL and CHI3L1 in the whole cohort. Note that each subgroup has the peak density of patients in a different position in reference with the median log values. (A) In non-MS control group, almost all patients fell in the left-inferior quadrant. (B) In PPMS subgroup, CHI3L1 levels were over the median in almost all patients, and two groups were distinguished with regards to NFL levels. (C) In the RRMS group, black triangles represented patients that at the end of the observation period evolved to progressive disease. (D) SPMS patients constitute an intermediate density map with three regions of distribution. NFL, neurofilament light chain; CHI3L1, chitinase-3-like-1; RRMS, relapsing-remitting multiple sclerosis; SPMS, secondary progressive multiple sclerosis; PPMS, primary progressive multiple sclerosis; Switch, diagnosis of SPMS during follow-up.

TABLE 3 | Multinomial regression model for combined NFL-CHI3L1value in clinical stage discrimination.

\begin{tabular}{|c|c|c|c|c|c|}
\hline & Estimate & Std. error & Exp (Estimate) & Lower $95 \%$ & Upper $95 \%$ \\
\hline PPMS Intercept ${ }^{a}$ & -1.552 & 0.34 & 0.212 & 0.102 & 0.394 \\
\hline SPMS Intercept & -0.819 & 0.239 & 0.441 & 0.267 & 0.697 \\
\hline Switch Intercept & -2.198 & 0.376 & 0.111 & 0.049 & 0.219 \\
\hline Non-MS controls Intercept & -7.931 & 2.44 & 0 & 0 & 0.018 \\
\hline PPMS logCHI3L1 & 1.644 & 0.388 & 5.173 & 2.514 & 11.199 \\
\hline SPMS logCHI3L1 & 0.804 & 0.311 & 2.235 & 1.233 & 4.181 \\
\hline SPMS logNFL & -0.948 & 0.34 & 0.387 & 0.195 & 0.723 \\
\hline SPMS logCHI3L1: logNFL & -0.896 & 0.399 & 0.408 & 0.177 & 0.819 \\
\hline Switch logCHI3L1 & 0.924 & 0.445 & 2.518 & 1.098 & 6.293 \\
\hline Switch logNFL & -0.064 & 0.419 & 0.938 & 0.415 & 2.129 \\
\hline
\end{tabular}

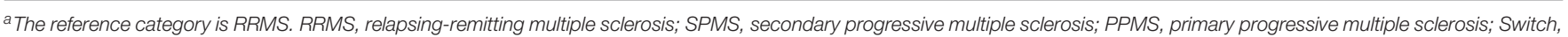

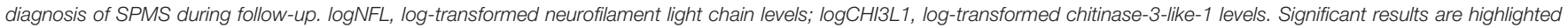
with bold letters.

Regarding the value of both CSF biomarkers in prognosis, recent reports in a CIS/RRMS cohort did not find a prognostic influence for these biomarkers in progression (43). Although we found that CSF NFL was predictive of disability accrual and prospective diagnosis of progressive disease in the bivariate analysis, it was not selected by the multivariable analysis as an 


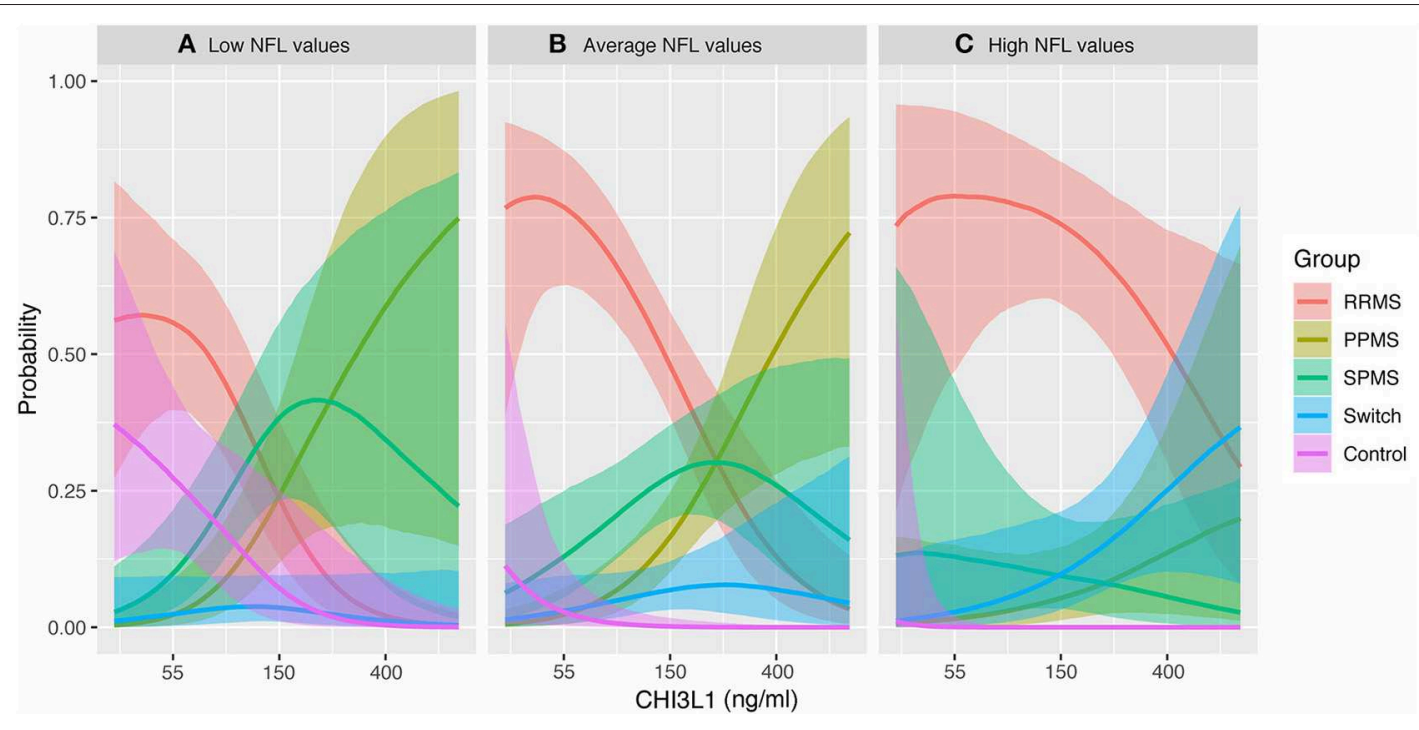

FIGURE 5 | Marginal effect plots. This plot is the graphical representation of the multinomial regression model performed and shows the probability to be comprised in a MS clinical form, including the probability of conversion to SPMS, with regards to CSF NFL, and CHI3L1 levels. Thus, this diagram shows the interaction between both biomarkers. (A) When levels of NFL are low, low CHI3L1 levels imply higher probability to be a non-MS control (pink) or a RRMS patient (red). High CHI3L1 levels in these patients are associated with the diagnosis of progressive MS (green and brownish green). Probability of switching to SPMS is very low. (B) With average levels of NFL, the probability of pertaining to a non-MS control decreases, and CHI3L1 levels discriminate better between RRMS (low CHI3L1), and PPMS (high CHI3L1). (C) With high NFL levels, the probability of being comprised in the RRMS group is the highest. High NFL and CHI3L1 are more probable in patients diagnosed of SPMS during follow-up, and in a subgroup of PPMS patients, likely PPMS patients with active disease. NFL, neurofilament light chain; CHI3L1, chitinase-3-like-1; RRMS, relapsing-remitting multiple sclerosis; SPMS, secondary progressive multiple sclerosis; PPMS, primary progressive multiple sclerosis; Switch, diagnosis of SPMS during follow-up.

independent predictor. In contrast, $\mathrm{CHI} 3 \mathrm{~L} 1$ alone was predictive of 1-point EDSS worsening and of the re-assignation of patients to progressive MS during follow-up. The median time for conversion to SPMS is normally $>10$ years as reported in natural history studies (44), and therefore, it is important to remark that our cohort was composed of MS patients with distinct clinical forms and at different time points from disease onset (with disease duration times up to 19 years), while the cohort studied by Sellebjerg et al. included only patients with recent diagnosis and who were followed for a median time of 5.7 years.

Further, we analyzed the usefulness of the combined measure of CSF NFL and CHI3L1 levels. Both values provided supplementary information of the MS phenotype with higher values of NFL that seemed to correspond more frequently to active patients (most in the RRMS subgroup) susceptible to respond to current DMT, while higher values of $\mathrm{CHI} 3 \mathrm{~L} 1$ were associated with progressive MS (more evident for PPMS). Eleven over a total of 14 RRMS patients diagnosed of SPMS during follow-up had high levels of both NFL and CHI3L1. This led us to investigate the combination of biomarkers and the ability to predict the diagnosis of progressive MS in patients from the RRMS cohort. We found that having high levels of CSF NFL and CHI3L1 was predictive of switching to the SPMS group. However, the number of patients that progressed during the study was low and CSF was collected at distinct time points from disease onset, hence we cannot infer from our data whether biomarkers could inform likeliness of conversion or were, indeed, detecting subclinical progression. Supporting the latter was the assessment of longer disease duration in these patients.

A disadvantage of measuring biomarkers in the CSF is the invasiveness nature of LP. This prevents for the use of CSF NFL and CHI3L1 levels in longitudinal studies. Currently, new detection methods allow precise detection of NFL in serum, and therefore allow for multiple measures during follow-up (12). Nevertheless, serum CHI3L1 levels are not specific of MS (45). In this report, we show that prognostic information can be obtained from a diagnostic lumbar puncture at any time from disease onset. Given the retrospective nature of the study, results should be interpreted cautiously, and our conclusions need to be confirmed by prospective analysis of larger cohorts. However, we would like to emphasize, as a strength of the study, that all patients had simultaneous assessment in CSF of NFL and CHI3L1 levels, OCMB, together with MRI and disability data, with a median follow-up of 50 months. The relationship of these biomarkers with OCMB and the value of the combined measure for MS profiling and recognition of progressive disease, as far as we know, had not yet been reported.

To conclude, individual measures of CSF NFL and CHI3L1 are biomarkers of disease activity and progression, respectively. The pattern of combined measure discriminates MS phenotypes and predicts the subset of RRMS patients that will progress clinically allowing early intervention. Whether early intervention guided by biomarkers can change the natural history of the disease is unknown, and should be the focus of future studies. 


\section{DATA AVAILABILITY}

The datasets generated for this study are available on request to the corresponding author.

\section{ETHICS STATEMENT}

All patients provided written informed consent. The study was approved by the Institutional Ethics Committee in Hospital Universitari I Politècnic La Fe (reference number PI17/01544).

\section{AUTHOR CONTRIBUTIONS}

SG-P and BC have designed the study, analyzed data, and wrote the manuscript. SG-P additionally carried out the data collection and statistical analysis. LC-N and JC-V have processed CSF and performed ELISA. BC has been attending physician and designed

\section{REFERENCES}

1. Bergman J, Dring A, Zetterberg H, Blennow K, Norgren N, Gilthorpe J, et al. Neurofilament light in CSF and serum is a sensitive marker for axonal white matter injury in MS. Neurol Neuroimmunol Neuroinflamm. (2016) 3:e271. doi: 10.1212/NXI.0000000000000271

2. Bonneh-Barkay D, Bissel SJ, Kofler J, Starkey A, Wang G, Wiley CA. Astrocyte and macrophage regulation of YKL-40 expression and cellular response in neuroinflammation. Brain Pathol. (2012) 22:530-46. doi: 10.1111/j.1750-3639.2011.00550.x

3. Blennow K, Hampel H, Weiner M, Zetterberg H. Cerebrospinal fluid and plasma biomarkers in Alzheimer disease. Nat Pub Group. (2010) 6:131-44. doi: $10.1038 /$ nrneurol.2010.4

4. Donker Kaat L, Meeter LH, Chiu WZ, Melhem S, Boon AJW, Blennow $\mathrm{K}$, et al. Serum neurofilament light chain in progressive supranuclear palsy. Parkinsonism Relat Disord. (2018) 56:98-101. doi: 10.1016/j.parkreldis.2018.06.018

5. Menke RAL, Gray E, Lu C-H, Kuhle J, Talbot K, Malaspina A, et al. CSF neurofilament light chain reflects corticospinal tract degeneration in ALS. Ann Clin Transl Neurol. (2015) 2:748-55. doi: 10.1002/acn3.212

6. Abdo WF, van de Warrenburg BPC, Munneke M, van Geel WJA, Bloem $\mathrm{BR}, \mathrm{Kremer} \mathrm{HPH}$, et al. CSF analysis differentiates multiple-system atrophy from idiopathic late-onset cerebellar ataxia. Neurology. (2006) 67:474-9. doi: 10.1212/01.wnl.0000227891.25592.8c

7. Sellebjerg F, Börnsen L, Ammitzbøll C, Nielsen JE, Vinther-Jensen T, Hjermind LE, et al. Defining active progressive multiple sclerosis. Mult Scler. (2017) 23:1727-35. doi: 10.1177/1352458517726592

8. Disanto G, Barro C, Benkert P, Naegelin Y, Schädelin S, Giardiello A, et al. Serum neurofilament light: a biomarker of neuronal damage in multiple sclerosis. Ann Neurol. (2017) 81:857-70. doi: 10.1002/ana.24954

9. Arrambide G, Espejo C, Eixarch H, Villar LM, Alvarez-Cermeño JC, Picón C, et al. Neurofilament light chain level is a weak risk factor for the development of MS. Neurology. (2016) 87:1076-84. doi: 10.1212/WNL.0000000000 003085

10. Håkansson I, Tisell A, Cassel $\mathrm{P}$, Blennow K, Zetterberg $\mathrm{H}$, Lundberg $\mathrm{P}$, et al. Neurofilament light chain in cerebrospinal fluid and prediction of disease activity in clinically isolated syndrome and relapsing-remitting multiple sclerosis. Eur J Neurol. (2017) 24:703-12. doi: 10.1111/ene.13274

11. Novakova L, Axelsson M, Malmeström C, Imberg H, Elias O, Zetterberg $\mathrm{H}$, et al. Searching for neurodegeneration in multiple sclerosis at clinical onset: diagnostic value of biomarkers. PLoS ONE. (2018) 13:e0194828. doi: 10.1371/journal.pone.0194828

12. Barro C, Benkert P, Disanto G, Tsagkas C, Amann M, Naegelin Y, et al. Serum neurofilament as a predictor of disease worsening and brain the database, the treatment protocol, and had been responsible with FP-M, FG, CA, and JD for the clinical follow-up. JG-M helped in collecting data. RG helped in designing figures and revising the manuscript. DH supervised the statistical analysis and helped in figure design.

\section{FUNDING}

This study was supported by the ISCIII (PI17/01544) and co-financed by the European Development Regional Fund. SG-P received a Rio Hortega fellowship from ISCIII (CM12/00014).

\section{ACKNOWLEDGMENTS}

We especially want to acknowledge Dr. Coret, co-founder and attending physician of the MS Unit in HCU, deceased last year. and spinal cord atrophy in multiple sclerosis. Brain. (2018) 141:2382-91. doi: 10.1093/brain/awy154

13. Kuhle J, Nourbakhsh B, Grant D, Morant S, Barro C, Yaldizli Ö, et al. Serum neurofilament is associated with progression of brain atrophy and disability in early MS. Neurology. (2017) 88:826-31. doi: 10.1212/WNL.0000000000003653

14. Piehl F, Kockum I, Khademi M, Blennow K, Lycke J, Zetterberg H, et al. Plasma neurofilament light chain levels in patients with MS switching from injectable therapies to fingolimod. Mult Scler. (2017) 24:1046-54. doi: $10.1177 / 1352458517715132$

15. Novakova L, Axelsson M, Khademi M, Zetterberg H, Blennow K, Malmeström $\mathrm{C}$, et al. Cerebrospinal fluid biomarkers as a measure of disease activity and treatment efficacy in relapsing-remitting multiple sclerosis. J. Neurochem. (2017) 141:296-304. doi: 10.1111/jnc.13881

16. de Flon P, Gunnarsson M, Laurell K, Söderström L, Birgander R, Lindqvist $\mathrm{T}$, et al. Reduced inflammation in relapsing-remitting multiple sclerosis after therapy switch to rituximab. Neurology. (2016) 87:141-7. doi: 10.1212/WNL.0000000000002832

17. Kuhle J, Plattner K, Bestwick JP, Lindberg RL, Ramagopalan SV, Norgren N, et al. A comparative study of CSF neurofilament light and heavy chain protein in MS. Mult Scler. (2013) 19:1597-603. doi: 10.1177/1352458513482374

18. Soelberg Sørensen P, Sellebjerg F. Neurofilament in CSF-A biomarker of disease activity and long-term prognosis in multiple sclerosis. Mult Scler. (2016) 22:1112-3. doi: 10.1177/1352458516658560

19. Mañé-Martínez MA, Olsson B, Bau L, Matas E, Cobo Calvo A, Andreasson U, et al. Glial and neuronal markers in cerebrospinal fluid in different types of multiple sclerosis. J Neuroimmunol. (2016) 299:112-7. doi: 10.1016/j.jneuroim.2016.08.004

20. Hinsinger G, Galéotti N, Nabholz N, Urbach S, Rigau V, Demattei C, et al. Chitinase 3-like proteins as diagnostic and prognostic biomarkers of multiple sclerosis. Mult Scler. (2015) 21:1251-61. doi: 10.1177/1352458514561906

21. Comabella M, Fernández M, Martin R, Rivera-Vallvé S, Borràs E, Chiva $\mathrm{C}$, et al. Cerebrospinal fluid chitinase 3-like 1 levels are associated with conversion to multiple sclerosis. Brain. (2010) 133:1082-93. doi: 10.1093/brain/awq035

22. Martínez MAM, Olsson B, Bau L, Matas E, Cobo Calvo A, Andreasson $\mathrm{U}$, et al. Glial and neuronal markers in cerebrospinal fluid predict progression in multiple sclerosis. Mult Scler. (2015) 21:550-61. doi: $10.1177 / 1352458514549397$

23. Modvig S, Degn M, Roed H, Sørensen TL, Larsson HBW, Langkilde AR, et al. Cerebrospinal fluid levels of chitinase 3-like 1 and neurofilament light chain predict multiple sclerosis development and disability after optic neuritis. Mult Scler. (2015) 21:1761-70. doi: 10.1177/1352458515574148

24. Cantó E, Reverter F, Morcillo-Suárez C, Matesanz F, Fernandez O, Izquierdo $\mathrm{G}$, et al. Chitinase 3-like 1 plasma levels are increased in patients with 
progressive forms of multiple sclerosis. Mult Scler. (2012) 18:983-90. doi: $10.1177 / 1352458511433063$

25. Quintana E, Coll C, Salavedra-Pont J, Muñoz-San Martín M, Robles-Cedeño $\mathrm{R}$, Tomàs-Roig J, et al. Cognitive impairment in early stages of multiple sclerosis is associated with high cerebrospinal fluid levels of chitinase 3like 1 and neurofilament light chain. Eur J Neurol. (2018) 25:1189-91. doi: $10.1111 /$ ene. 13687

26. Malmeström C, Axelsson $M$, Lycke J, Zetterberg $H$, Blennow $K$, Olsson B. CSF levels of YKL-40 are increased in MS and replaces with immunosuppressive treatment. J Neuroimmunol. (2014) 269:87-9. doi: 10.1016/j.jneuroim.2014.02.004

27. Matute-Blanch C, Río J, Villar LM, Midaglia L, Malhotra S, Alvarez-Cermeño JC, et al. Chitinase 3-like 1 is associated with the response to interferonbeta treatment in multiple sclerosis. J Neuroimmunol. (2017) 303:62-5. doi: 10.1016/j.jneuroim.2016.12.006

28. Novakova L, Axelsson M, Khademi M, Zetterberg H, Blennow K, Malmeström $\mathrm{C}$, et al. Cerebrospinal fluid biomarkers of inflammation and degeneration as measures of fingolimod efficacy in multiple sclerosis. Mult Scler. (2017) 23:62-71. doi: 10.1177/1352458516639384

29. Villar LM, Masjuan J, González-Porqué P, Plaza J, Sádaba MC, Roldán E, et al. Intrathecal IgM synthesis predicts the onset of new relapses and a worse disease course in MS. Neurology. (2002) 59:555-9. doi: 10.1212/WNL.59.4.555

30. Villar LM, Masjuan J, Gonzalez-Porque P, Plaza J, Sadaba MC, Roldán E, et al. Intrathecal IgM synthesis is a prognostic factor in multiple sclerosis. Ann Neurol. (2003) 53:222-6. doi: 10.1002/ana.10441

31. Villar LM, Sadaba MC, Roldán E, Masjuan J, Gonzalez-Porque P, Villarrubia $\mathrm{N}$, et al. Intrathecal synthesis of oligoclonal IgM against myelin lipids predicts an aggressive disease course in MS. J Clin Invest. (2005) 115:187-94. doi: 10.1172/JCI200522833

32. McNicholas N, Hutchinson M, McGuigan C, Chataway J. 2017 McDonald diagnostic criteria: a review of the evidence. Mult Scler Relat Disord. (2018) 24:48-54. doi: 10.1016/j.msard.2018.05.011

33. Lublin FD, Reingold SC, Cohen JA, Cutter GR, Sørensen PS, Thompson AJ, et al. Defining the clinical course of multiple sclerosis: the 2013 revisions. Neurology. (2014) 83:278-86. doi: 10.1212/WNL.0000000000000560

34. Thompson AJ, Banwell BL, Barkhof F, Carroll WM, Coetzee T, Comi G, et al. Diagnosis of multiple sclerosis: 2017 revisions of the McDonald criteria. Lancet Neurol. (2018) 17:162-73. doi: 10.1016/S1474-4422(17)30470-2

35. Kurtzke JF. Rating neurologic impairment in multiple sclerosis: an expanded disability status scale (EDSS). Neurology. (1983) 33:1444-52. doi: 10.1212/WNL.33.11.1444

36. Rotstein DL, Healy BC, Malik MT, Chitnis T, Weiner HL. Evaluation of no evidence of disease activity in a 7-year longitudinal multiple sclerosis cohort. JAMA Neurol. (2015) 72:152-8. doi: 10.1001/jamaneurol.2014.3537
37. Teunissen CE, Dijkstra C, Polman C. Biological markers in CSF and blood for axonal degeneration in multiple sclerosis. Lancet Neurol. (2005) 4:32-41. doi: 10.1016/S1474-4422(04)00964-0

38. Khademi M, Dring AM, Gilthorpe JD, Wuolikainen A, Nimer Al F, Harris $\mathrm{RA}$, et al. Intense inflammation and nerve damage in early multiple sclerosis subsides at older age: a reflection by cerebrospinal fluid biomarkers. PLoS ONE. (2013) 8:e63172. doi: 10.1371/journal.pone.0063172

39. Khalil M, Teunissen CE, Otto M, Piehl F, Sormani MP, Gattringer T, et al. Neurofilaments as biomarkers in neurological disorders. Nat Rev Neurol. (2018) 14:577-89. doi: 10.1038/s41582-018-0058-Z

40. Burman J, Raininko R, Blennow K, Zetterberg H, Axelsson M, Malmeström C. YKL-40 is a CSF biomarker of intrathecal inflammation in secondary progressive multiple sclerosis. J Neuroimmunol. (2016) 292:52-7. doi: 10.1016/j.jneuroim.2016.01.013

41. Correale J, Fiol M. Chitinase effects on immune cell response in neuromyelitis optica and multiple sclerosis. Mult Scler. (2011) 17:521-31. doi: $10.1177 / 1352458510392619$

42. Norden DM, Godbout JP. Review: microglia of the aged brain: primed to be activated and resistant to regulation. Neuropathol Appl Neurobiol. (2013) 39:19-34. doi: 10.1111/j.1365-2990.2012.01306.x

43. Sellebjerg F, Royen L, Soelberg Sørensen P, Oturai AB, Jensen PEH. Prognostic value of cerebrospinal fluid neurofilament light chain and chitinase-3-like1 in newly diagnosed patients with multiple sclerosis. Mult Scler. (2018). doi: 10.1177/1352458518794308. [Epub ahead of print].

44. Koch M, Kingwell E, Rieckmann P, Tremlett H, UBC MS Clinic neurologists. The natural history of secondary progressive multiple sclerosis. J Neurol Neurosurg Psychiatry. (2010) 81:1039-43. doi: 10.1136/jnnp.2010. 208173

45. Schultz NA, Johansen JS. YKL-40-A protein in the field of translational medicine: a role as a biomarker in cancer patients? Cancers. (2010) 2:1453-91. doi: $10.3390 /$ cancers 2031453

Conflict of Interest Statement: The authors declare that the research was conducted in the absence of any commercial or financial relationships that could be construed as a potential conflict of interest.

Copyright (c) 2019 Gil-Perotin, Castillo-Villalba, Cubas-Nuñez, Gasque, Hervas, Gomez-Mateu, Alcala, Perez-Miralles, Gascon, Dominguez and Casanova. This is an open-access article distributed under the terms of the Creative Commons Attribution License (CC BY). The use, distribution or reproduction in other forums is permitted, provided the original author(s) and the copyright owner(s) are credited and that the original publication in this journal is cited, in accordance with accepted academic practice. No use, distribution or reproduction is permitted which does not comply with these terms. 